\title{
33rd ANNUAL SASKATCHEWAN CHRISTMAS BIRD COUNT, 1974
}

\author{
Compiled by MARY I. HOUSTON*
}

The exceptionally mild winter weather up to and including the Christmas Count period is perhaps responsible for the large numbers of species of waterfowl recorded. Six species of birds were added this year to the all time list, five of these being waterfowl - Snow Goose and Greater Scaup being reported from Regina; White-fronted Goose and Greenwinged Teal (previously on the additional list) from Saskatoon; and White-winged Scoter from Cold River. The other new species was a Whitecrowned Sparrow from Saskatoon. This brings the all-time total to 122 species seen on Count days since counts began in 1942 and an additional 6 species seen during the count period, but not on count days.

The unusual weather brought out more counters for longer periods of time and provided a more thorough coverage of the areas, which helps to account for the record high number of species seen in any 1 year: 79 species plus 4 additionals.

Many comments were made, however, about the lack in numbers of birds, particularly the winter birds such as Snow Buntings, Bohemian Waxwings and Pine Grosbeaks.

Most interesting was the number of localities reporting Golden-crowned Kinglets (13 including one locality where birds were seen as additional). This species has been recorded in only 11 of the past 33 years and never as widely spread or in such large numbers.

Gray Partridge, reported from only 14 localities, represented the major decrease.

* 863 University Drive,

Saskatoon, Satsk.
Counters from 44 localities (see map) participated in Bird Counts this year.

The map was prepared by Wayne Harris.

1. BANGOR. Dec. 29; around yard and farm: 8 species, 26 individuals. - Mrs. A. Thompson.

2. BATTLEFORD. Dec. 26:: 3 miles on foot in 2 hours and 73 miles by car in 4 hours, tcmp. $32^{\circ}$, calm, 3 inchcs of snow; 13 specics, 479 individuals. - S. G. Sealy.

3. BESNARD LAKE $\left(55^{\circ} 20^{\prime} \mathrm{N} 106^{\circ}\right.$ $\left.00^{\prime} \mathrm{W}\right)$. Dec. 27: 25 miles on foot, 18 on skis, 14 on snowshoes, 10 by car, in $25-1 / 2$ party hours, tcmp. $16^{\circ}-30^{\circ}$, no wind, sunny in A.M. clouding over in P.M., 4 in. snow; 12 specics, 339 individuals. (Add: Blackbilled Magpie, 1, Dec. 26; Northern Shrike, 1. Dec. 26). - Betty, John, Jon (compiler), Naomi, Nikki, Pauline and Peter Gerrard.'

4. BIGGAR. Dec. 23; 75 miles by car in 6-1/2 hours, 6 miles on foot in 6 hours, temp. $13^{\circ}$, wind $0-4 \mathrm{mph}$, overcast in A.M. clear in P.M., about 1 inch of snow; 14 spccies, 1033 individuals. (Add: Roughlegged Hawk, 1, Dec. 15, 20, 25, 27 and Jan. 1: Amcrican Mcrlin, 1, Dec. 15, 20, Jan. 2: Northern Shrike, 1, Dec. 25). Don Renaud, Wayne Renaud, Alan Smith, Guy Wapple (compiler), Rodney Wapple.

5. BIG GULLY CREEK ( 15 miles NE of Maidstone). Dec. 19; 15 miles by car in 1 hour and 10 miles on foot in 5 hours, temp. $0^{\circ}-15^{\circ}$, cloudy in A.M., clear in P.M., wind N $0-5$ mph, 1/2 inch of snow; 15 species, 99 individuals. - Wayne Harris, Sheila Lamont.

6. BIRD'S POINT (on Round Lake, 7 miles S and 3 miles W of Stockholm). Dec. 29; at yard fecder; 7 species, 44 individuals. - Doug Francis.

7. BORDEN. Dec. 28: 2 miles on foot in 2 hours and 92 miles by car in $6-1 / 2$ hours, temp. $11^{\circ}-24^{\circ}$, wind $\mathrm{W} 7-11 \mathrm{mph}, 2$ inches of snow; 10 species, 764 individuals. Mark Abley, John Shadick, Stan Shadick (compiler). 


\begin{tabular}{|c|c|c|c|c|c|c|c|c|c|c|c|}
\hline & 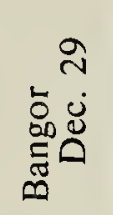 & 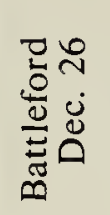 & 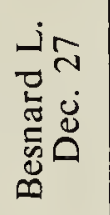 & 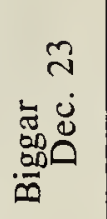 & $\begin{array}{l}20 \\
\overline{\bar{z}} \\
0 \\
0 \\
. \infty \\
0\end{array}$ & 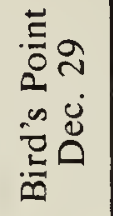 & 离 & 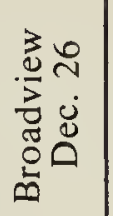 & 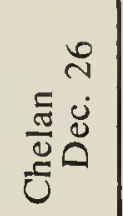 & 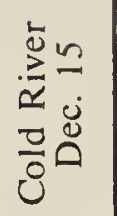 & 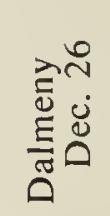 \\
\hline Mallard & & & & & & & & & & 73 & \\
\hline \multicolumn{12}{|l|}{ Pintail } \\
\hline \multicolumn{12}{|l|}{ Redhead } \\
\hline \multicolumn{12}{|l|}{ Lesser Scaup } \\
\hline Common Goldeneye & & & & & & & & 1 & & 26 & \\
\hline Goshawk & & & 1 & 1 & & & & & & & 1 \\
\hline Rough-leg. Hawk & & 1 & & & & & & & & & \\
\hline Golden Eagle & & & & & & & & 1 & & & \\
\hline Bald Eagle & & & & & & & & & & 1 & \\
\hline \multicolumn{12}{|l|}{ Merlin } \\
\hline Spruce Grouse & & & 2 & & & & & & & & \\
\hline Ruffed Grouse & & 2 & & & 3 & & & 2 & & 1 & \\
\hline Sharp-tailed Grouse & 8 & 1 & & 28 & 7 & & 22 & & 2 & & 19 \\
\hline \multicolumn{12}{|l|}{ Ring-neck. Pheasant } \\
\hline Gray Partridge & & 8 & & & & & 14 & & & & 20 \\
\hline Rock Dove & & 1 & & 55 & & & 10 & & & & 6 \\
\hline \multicolumn{12}{|l|}{ Mourning Dove } \\
\hline Great Horned Owl & & & & 2 & 1 & & 2 & 2 & & & 3 \\
\hline Snowy Owl & 1 & & & 6 & & & & & 1 & & \\
\hline \multicolumn{12}{|l|}{ Short-eared Owl } \\
\hline \multicolumn{12}{|l|}{ Common Flicker } \\
\hline Pileated Woodpecker & & & 1 & & & & & & & & \\
\hline Hairy Woodpecker & 1 & & 4 & & 2 & 1 & & 3 & & & 1 \\
\hline Downy Woodpecker & 2 & & 1 & 1 & 3 & 1 & & 2 & & & \\
\hline Horned Lark & & & & 6 & & & 7 & & & & 2 \\
\hline Gray Jay & & & 67 & & & & & & & & \\
\hline Blue Jay & & & & & 2 & & & 1 & & & \\
\hline Black-billed Magpie & 2 & 32 & & 29 & 13 & 2 & 47 & 12 & 2 & 5 & 39 \\
\hline Common Raven & & & 195 & & & & & & 3 & 7 & \\
\hline Bl.-cap. Chickadee & 4 & 6 & 22 & 13 & 1 & 4 & 22 & 5 & 4 & & 10 \\
\hline Boreal Chickadee & & & 31 & & 2 & & & & & & \\
\hline W-breasted Nuthateh & & & & & & 2 & & 2 & & & \\
\hline R-breasted Nuthatch & 1 & & & & & & & & & & \\
\hline \multicolumn{12}{|l|}{ Brown Creeper } \\
\hline \multicolumn{12}{|l|}{ American Robin } \\
\hline Gold.-cr. Kinglet & & & & & 45 & & & 12 & & & \\
\hline Bohemian Waxwing & & 399 & & 133 & & & 23 & 2 & & & 15 \\
\hline \multicolumn{12}{|l|}{ Cedar Waxwing } \\
\hline \multicolumn{12}{|l|}{ Northern Shrike } \\
\hline Starling & & 1 & & 1 & & & & & & & 1 \\
\hline House Sparrow & 7 & 10 & & 742 & 10 & 4 & 480 & 168 & 50 & & 128 \\
\hline \multicolumn{12}{|l|}{ West. Meadowlark } \\
\hline \multicolumn{12}{|l|}{ Rusty Blackbird } \\
\hline Evening Grosbeak & & & & & & 30 & & 16 & & & \\
\hline Pine Grosbeak & & 4 & & & 4 & & & & & & 5 \\
\hline Hoary Redpoll & & & 12 & & & & & & & & \\
\hline Common Redpoll & & 13 & & 14 & 1 & & 137 & 72 & & & 20 \\
\hline \multicolumn{12}{|l|}{ Red Crossbill } \\
\hline \multicolumn{12}{|l|}{ Dark-eyed Junco } \\
\hline Snow Bunting & & 1 & & 2 & & & & 500 & & 38 & 80 \\
\hline & & & & & & & & & & & \\
\hline Single Species & & & 2 & & 2 & & & 2 & & 1 & \\
\hline
\end{tabular}

Species recorded from more than 1 locality on Count day 


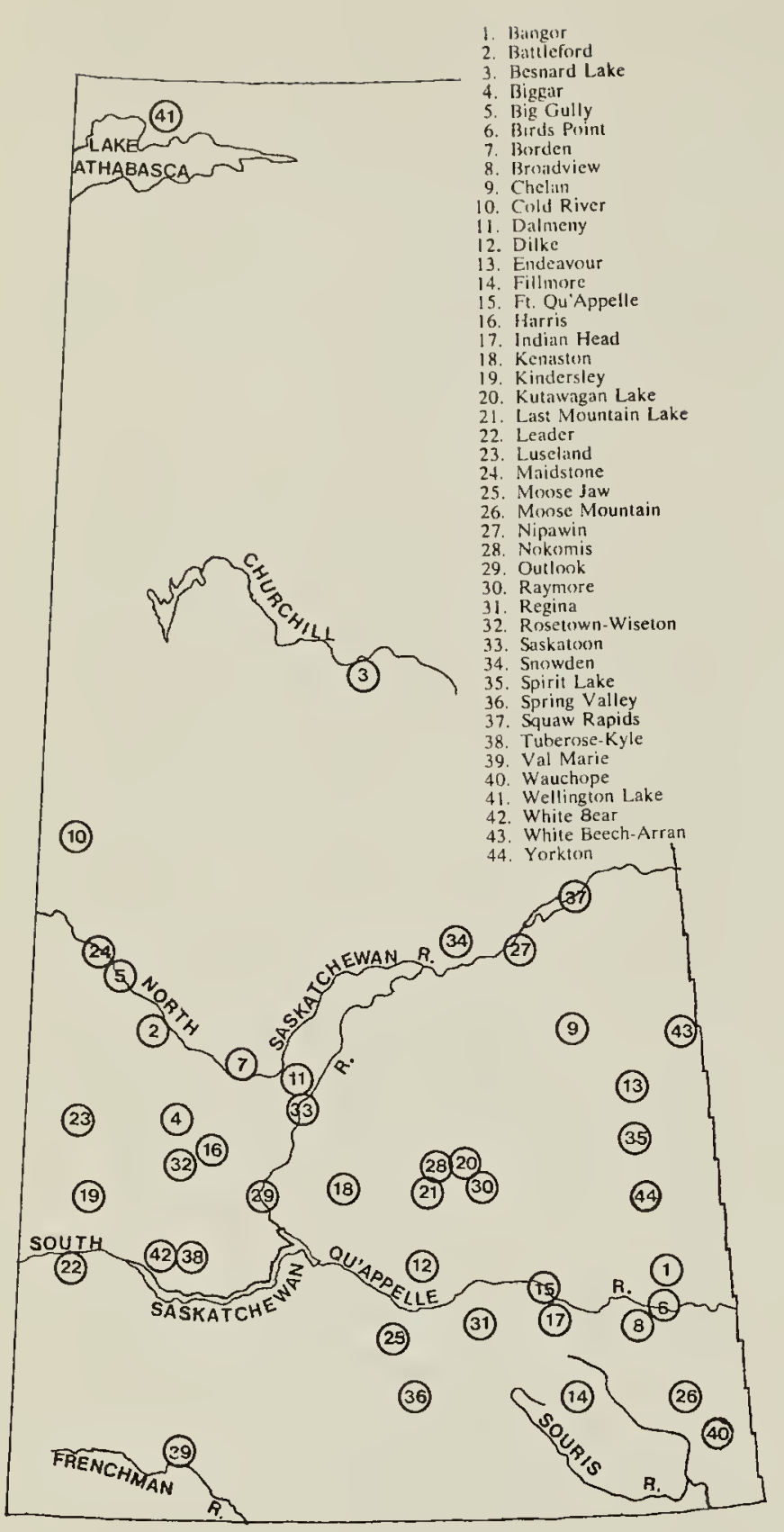

8. BROADVIEW. Dec. 26; 120 miles by car in $7-1 / 2$ hours, $2-1 / 2$ miles on foot, temp. $29^{\circ}$, wind light: 18 species, 804 individuals. (Add: Sharp-tailed Grouse, 18, Dec. 24; Rock Dove, 50, Dec. 24; Horned Lark, 3, Dec. 24: Northern Shrike, 1, Dec. 24: Pine Grosbeak, 30, Dec. 25). - David Chaskavich, Don Weidl.

9. CHELAN. Dec. 26; 1 mile on foot, 5 miles on ski-doo, temp. $28^{\circ}-32^{\circ}$, clear, calm, 4 inches of snow: 6 species, 62 individuals. - Joy Aspen, Keith Aspen, Lane Harris, Tim Harris.

10. COLD RIVER $\left(54^{\circ} 33^{\prime} \mathrm{N}, 109^{\circ} 50^{\prime} \mathrm{W}\right)$. Dec. 15: 15 miles by car and 3 miles on foot, temp. $20^{\circ}$, overcast, calm: 8 species, 153 individuals. - William Haras.

11. DALMENY. Dec. 26; 41-1/2 miles on foot in $21-1 / 4$ hours, temp. $15^{\circ}-26^{\circ}$, wind W changing to SSE, cloudy periods; 15 species, 350 individuals. (Add: Ruffed Grouse, 1, Dec. 31) - Mara Johanson, Brian Sperling, Gilbert Sperling (compiler), Lloyd Sperling.
12. DILKE. Dec. $29 ; 78$ miles by car, 3 miles on foot in $6-1 / 4$ hours, temp. $10^{\circ}-20^{\circ}$ wind SW 5-20 mph: 5 species, 332 individuals. (Add: Rough-legged Hawk, 1 Dec. 27; American Merlin, 1, Dec. 27 Snowy Owl, 1, Dec. 20, 28). - J. B Belcher, Margaret Belcher, Mr. and Mrs. S R. Belcher, Stan Houston, Ken McArton Peg Mortin.

13. ENDEAVOUR. Dec. 21 ; around farm yard, temp. $10^{\circ}$, overcast, calm; 8 species 150 individuals. (Add: Ruffed Grouse, 1, Dec. 23; Snowy Owl, 1, Dec. 24; Downy Woodpecker, 1, Dec. 23; Gray Jay, 1, Dec. 24; Evening Grosbeak, 5, Dec. 25; Common Redpoll, 58, Dec. 27). - William Haras.

14. FILLMORE. Dec. 26; 21 miles by car in $1-1 / 2$ hours, 1 mile on foot in 1 hour around 2 yards $3 / 4$ hour, temp. $22^{\circ}$, sunny wind light, 10 inches of snow: 7 species 296 individuals. (Add: Golden Eagle, 1 Dec. 24; Snow Bunting, 260, Dec. 24). Flossie Bogdan (compiler), Larry Wiggins Marie Wiggins, Michael Wiggins, Michelle Wiggins.

15. FORT QU'APPELLE. Dec. 28:30 miles by car and 1 mile on foot in $3-1 / 2$ hours, $3-1 / 2$ miles on skis in 2 hours, temp. $25^{\circ}$, wind light, partly cloudy, $3-1 / 2$ inches of snow; 4 species, 91 individuals. (Add: Mallard, 1, Dec. 15, 18, Dec. 16, 2, Dec. 26, 3, Dec. 30; Common Goldeneye, '3I, Dec. 16, 4, Dec. 25: Ruffed Grouse, 3, Dec. 25; Snowy Owl, 1, Dec. 19; Downy Woodpecker, 1, Dec. 18; Blue Jay, 1, Dec. 15). Arthur Bredalsen, Mrs. Jenny Gray, Inez and Joe Kralkay, Mrs. Dorothy Williams, Kay de Vries (compiler), Bernard de Vries. (Members and Guests of the Fort Qu'Appelle Naturalists' Society.)

16. HARRIS. Dec. 15;31-1/2 miles on foot in 15 hours, 290 miles by car in $18-1 / 2$ hours, temp. $20^{\circ}-23^{\circ}$, overcast, wind NW 0$8 \mathrm{mph}$, no snow; 26 species, 2149 individuals. - Bob Godwin, Wayne Harris, Mary and Stuart Houston, Sheila Lamont, Ken Lumbis, Kathy Meers, John Polson, Don Renaud (compiler), Guy Wapple, Jim and Shirley Wedgwood.

17. INDIAN HEAD. Dec. 29; 35 miles by car in 3 hours, $4-1 / 2$ miles on foot in 4 hours, temp. $20^{\circ}-30^{\circ}$, calm, sunny, 8 inches of snow; 20 species, 1417 individuals. (Add: Peregrine Falcon, I, Dec. 23, 27; Sharp-tailed Grouse, 50, Dec. 27; Ringnecked Pheasant, 1, Dec. 26; Mourning Dove, 2, Dec. 23; Long-eared Owl, 1, Jan. 1: Horned Lark, 6, Dec. 28; Blue Jay, 1 Dec. 28; Golden-crowned Kinglet, 6, Dec 28: Common Redpoll, 50, Dec. 26.) Betty and Cec Ashmore, Peter and Therese 
Species recorded frop more than 1 locality on Count day

Mallard

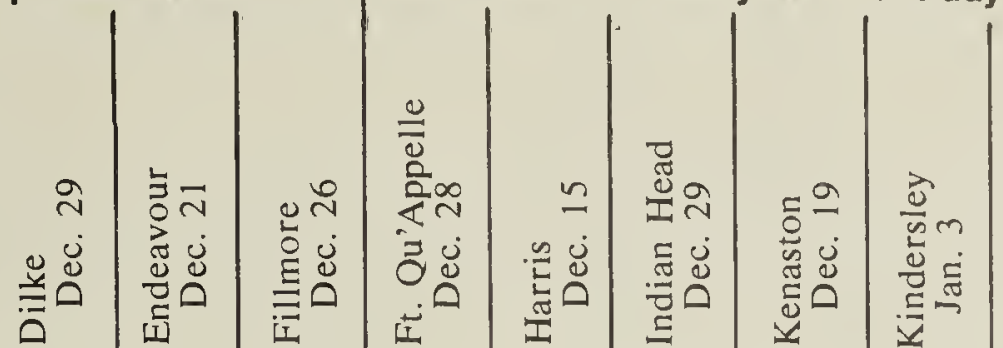

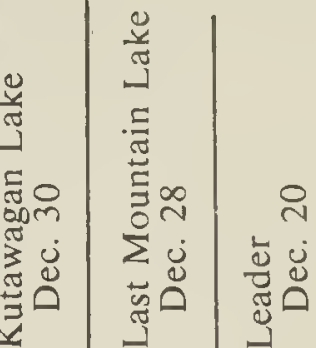

Pintail

Redhead

Lesser Scaup

Common Goldeneye

Goshawk

Rough-leg. Hawk

Golden Eagle

Bald Eagle

Merlin

Spruce Grouse

Ruffed Grouse

Sharp-tailed Grouse

Ring-neck. Pheasant

Gray Partridge

Rock Dove

Mourning Dove

Great Horned Owl

Snowy Owl

Short-eared Owl

Common Flicker

Pileated Woodpecker

Hairy Woodpecker

Downy Woodpecker

Horned Lark

Gray Jay

Blue Jay

Black-billed Magpie

Common Raven

Bl.-cap. Chickadee

Boreal Chickadee

W-breasted Nuthatch

R-breasted Nuthatch

Brown Creeper

American Robin

Gold.-cr. Kinglet

Bohemian Waxwing

Cedar Waxwing

Northern Shrike

Starling

House Sparrow

West. Meadowlark

Rusty Blackbird

Evening Grosbeak

Pine Grosbeak

Hoary Redpoll

Common Redpoll

Red Crossbill

Dark-eyed Junco

Snow Bunting

Single Species

150

\begin{tabular}{|r|r|r|r|r|r|r|r|r|r|r|}
\hline & & & & & & & & 1 & & \\
\hline & & & & 1 & & & & & & \\
\hline & & & & 1 & & & & & & \\
\hline & 12 & 15 & & 180 & & 9 & & & 12 & \\
\hline & & & & & & & & & & \\
\hline
\end{tabular}

March, 1975. 33(1) 
Species recorded from more than 1 locality on Count day

\begin{tabular}{|c|c|c|c|c|c|c|c|c|c|c|c|}
\hline & & & & & & & & & 500 & & 14 \\
\hline $\begin{array}{l}\text { Mallard } \\
\text { Pintail } \\
\end{array}$ & & & & & & & & & 1 & & 3 \\
\hline Pintail & & & & & & & & & 3 & & \\
\hline Lesser Scaup & & & & & & & & & 9 & & 8 \\
\hline Common Goldeneye & & & & & & & & & 8 & & 130 \\
\hline Goshawk & & & & & & & & 1 & & & 3 \\
\hline Rough-leg. Hawk & & & & & & & & & & & 1 \\
\hline Golden Eagle & & & & & & & & & & & 1 \\
\hline Bald Eagle & & 1 & & & & & & & & & \\
\hline Merlin & & & & & & 1 & & & 4 & & 6 \\
\hline Spruce Grouse & & & & & & & & & & & \\
\hline Ruffed Grouse & & 2 & & 1 & 1 & & & 1 & & & 6 \\
\hline Sharp-tailed Grouse & 9 & 4 & 2 & & & 3 & & 11 & 7 & & 59 \\
\hline Ring-neck. Pheasant & & & 14 & & & & & & & & 10 \\
\hline Gray Partridge & 9 & & & & & & & & 47 & 19 & 57 \\
\hline Rock Dove & 4 & 1 & 194 & & 5 & & 17 & 140 & 360 & 6 & 805 \\
\hline Mourning Dove & & & & & & & & & & & 1 \\
\hline Great Horned Owl & 1 & 1 & 1 & & & & 1 & 2 & 1 & & 6 \\
\hline Snowy Owl & 3 & & 3 & & & 1 & 2 & 1 & 10 & 2 & 11 \\
\hline Short-eared Owl & & & & & & 3 & & & 1 & & \\
\hline Common Flicker & & & & & & & & & & & 1 \\
\hline Pileated Woodpecker & & & & & & & & & & & \\
\hline Hairy Woodpecker & & 3 & & 5 & 2 & & & 1 & & & 4 \\
\hline Downy Woodpecker & 1 & 1 & & 2 & 1 & & & 1 & 1 & & 10 \\
\hline Horned Lark & 3 & & 1 & & & & & 2 & & 3 & 6 \\
\hline Gray Jay & & & & & & & & & & & \\
\hline Blue Jay & & 1 & & 11 & 1 & & & & & & 15 \\
\hline Black-billed Magpie & 60 & 27 & 71 & 12 & 12 & 18 & 2 & 95 & 53 & 7 & 381 \\
\hline Common Raven & & 1 & & & 29 & & & & & & \\
\hline Bl.-cap. Chickadee & 1 & 6 & 2 & 7 & 3 & & & 11 & 2 & & 109 \\
\hline Boreal Chickadee & & & & & 2 & & & & & & 2 \\
\hline W-breasted Nuthatch & & & & 1 & & & & & & & \\
\hline R-breasted Nuthatch & & & & 9 & & & & & 11 & & 8 \\
\hline Brown Creeper & & & & & & & & & 3 & & \\
\hline American Robin & & & & & & & & & & & 1 \\
\hline Gold.-cr. Kinglet & 1 & & 6 & 2 & & & & & 13 & & 10 \\
\hline Bohemian Waxwing & 114 & & 3 & & 2 & 1 & & 69 & & & 887 \\
\hline Cedar Waxwing & & & 5 & & & & & & & & 59 \\
\hline Northern Shrike & & 1 & & & & & & & 3 & & \\
\hline Starling & & & 21 & & & & & & 115 & 10 & \begin{tabular}{|l|}
51 \\
\end{tabular} \\
\hline House Sparrow & 163 & 20 & 920 & & 20 & 103 & 6 & 1770 & 4132 & 150 & \begin{tabular}{|l|}
2796 \\
\end{tabular} \\
\hline West. Meadowlark & 1 & & & & & & & & 1 & & \\
\hline Rusty Blackbird & & & & & & & & & 5 & & \\
\hline Evening Grosbeak & & & & & 81 & & & 2 & & & 1 \\
\hline Pine Grosbeak & & 1 & 6 & 17 & 4 & & & & & & 1 \\
\hline Hoary Redpoll & 1 & 1 & & & & & & & & & \\
\hline Common Redpoll & 82 & 335 & 60 & 2 & 96 & & & & 45 & & 285 \\
\hline Red Crossbill & & & & & & & & & & & 4 \\
\hline Dark-eyed Junco & & & & & & & & & & & 3 \\
\hline Snow Bunting & 127 & 839 & 1 & & & 50 & 10 & 58 & 1554 & 180 & 160 \\
\hline & & & & & & & & & & & \\
\hline Single Species & & & & & & & & & 13 & & \\
\hline
\end{tabular}


rrett, Carol and Marg Beaulieu, Ernie d Hazel Buglass, Helen King, Rose Laughlin, Lorne Scott, Ken Skinner, ry Skinner (compiler).

KENASTON. Dec. 19; temp. 25, nny, calm, 2 inches of snow: 11 species, 0 individuals. (Add: Great Horned Owl, c. 21; Snowy Owl, Dec. 20, 28, 29; Snow nting, 500, Dec. 20). - P. Lawrence ckie.

KINDERSLEY. Jan. 3; 10 miles by , $1 / 4$ mile on foot, temp. $28^{\circ}$, clear, Im, I inch of snow; 5 species, 157 inviduals. - Jean M. Harris.

KUTAWAGAN LAKE (centered 12 les north of Semans). Dec. 30; 55 miles $\mathrm{ca}_{i}$ in 5 hours and 5-1/2 miles on foot in hours, temp. $12^{\circ}-27^{\circ}$, overcast, wind $\mathrm{S}$ to 5-10 mph., 4 inches of snow; 8 species, 9 individuals. (Add: Canada Goose, 1, ec. 20; Snowy Owl, 1, Dec. 23). - Duane d Ken Harding, Wayne Harris, Sheila mont.

\section{LAST MOUNTAIN LAKE} hanagement unit and immediate area). ec. 28; 103 miles by car in 5 hours and 6 iles on foot in 2 hours, temp. $16^{\circ}-20^{\circ}$, nny, wind WSW 10 to $25 \mathrm{mph}, 4$ inches snow; 8 species, 564 individuals. (Add: ugh-legged Hawk, 1, Dec. 27; Starling, Dec. 27). - Greta Harris, Wayne arris, Sheila Lamont.

LEADER. Dec. 20; 9 miles by car, d on foot about farm for 3 hours, temp. , clear, calm, no snow; 7 species, 33 inviduals. - Daisy Meyers.

LUSELAND. Jan. 1; 40 miles by car in ours, 5 miles on foot in 5 hours, temp. $-32^{\circ}, 50 \%$ high overcast, calm, 2 inches snow; 16 species, 580 individuals. rry Finley, Kim Finley, Bill Holton, ayne Renaud.

MAIDSTONE FERRY (21 miles north Maidstone). Dec. 24; 60 miles by car in 3 urs and 7 miles on foot in 3 hours, temp. to $21^{\circ}$, cloudy, wind S to NW $10 \mathrm{mph}, 6$ hes of snow; 17 species, 1245 iniduals. (Add: Horned Lark, 4, Dec. 22). Wayne Harris, Sheila Lamont.

MOOSE JAW. Dec. 26; 121 miles by and 3 miles on foot, temp. $29^{\circ}$, sunny, nd W $10 \mathrm{mph}, 2$ inches of snow; 16 ecies, 1310 individuals. - Jeanette and ane Fjetland, Doug Francis, Ruth Hill, t Kern, Leith and Cy Knight, Moray wis.

MOOSE MOUNTAIN. Dec. 29; 65 les by car in 5 hours, 2-1/2 miles on foot, mp. $20^{\circ}$, clear, calm; 11 species, 69 inviduals. - David Chaskavich, Don eidl.
27. NIPAWIN. Dec. 27; 116 miles in 5 hours by car, 4 hours on foot, temp. $30^{\circ}$, mostly clear; 14 species, 259 individuals. - David Riome, Stan Riome.

28. NOKOMIS. Dec. 30; $56 \mathrm{mi}$. by car in 3 hours and 10 minutes; temp. $15^{\circ}-28^{\circ}$, sunny then light overcast, very little snow; 8 species, 180 individuals. - Jim Hamilton (compiler), Jack Woods.

29. OUTLOOK. Dec. 26; temp. $20^{\circ}$, wind SE, 1 inch of snow: 6 species, 38 individuals. (Add: Mallard, 21, Dec. 27; Common Goldeneye, 2, Dec. 27). Harold Kvinge.

30. RAYMORE. Dec. 29; 136 miles by car in 6 hours and 10-1/2 miles on foot in 5-1/2 hours, I hour at feeder, temp. $0^{\circ}-16^{\circ}$, clear, wind SW 10-25 mph, 4-1/2 inches of snow: 15 species, 2165 individuals. (Add: Roughlegged Hawk, 1, Dec. 23; Northern Shrike, 1, Dec. 24; Common Redpoll, 10, Dec. 27 and 75, Dec. 30). - Chas., Greta and Wayne Harris, Sheila Lamont.

31. REGINA. Dec. $26 ; 607$ miles by car in 48 hours, 43 miles on foot in 25 hours, 8 hours at feeders, temp. $16^{\circ}-25^{\circ}$, clear, W wind $10 \mathrm{mph}$, snow $0-20$ inches; 38 species, 7779 individuals. (Add: Gadwall, 1: Bluewinged Teal, 2; American Wigeon, 1; Bufflehead, 1: Common Flicker, 1; Hairy Woodpecker, 1; Horned Lark, 7; American Robin, 5; Bohemian Waxwing, 9; Cedar Waxwing, 4; Evening Grosbeak, 1; Pine Grosbeak, 2; White-winged Crossbill, 1; Dark-eyed Junco, 3). - Joan Ackerman, Gary Anweiler, Jessie Bailey, Joan Baron, Gordon Barr, Keith Barr, Margaret Belcher, Rev. Tom Beveridge, Mrs. T. Beveridge, Al Binnie, Betty Binnie, Greg Bobbitt, Robbie Boychuk, Frank Brazier, Eric Cooke, Betty Cruickshank, Robyn Donison, Lucy Eley, George Ferguson, Wayne Gemmell, Jan Harrigan, Al Harrison, Val Harrison, Jim Hines, Rose Hines, Melissa Jackson, Jim Jowsey, Shirley Jowsey, Elizabeth and Ernie Kassian, Darlene Kauk, Shirley Larmour, George Ledingham, K. Leibel, Bob Luterbach, Pat MacKenzie, Joe Miller, Will Marchuk, Ken Mayer, Madeleine McGinnis, George Mitchell, Helen Morrison, Frank Nargang, Dave Phillips, Connie Pratt, Brian Rainey, Joe Roberts, Carole Spencer, John Spencer, Rick St. Pierre, Frank Switzer, Bob Tegart, Dorothy Tegart, Les Toews, Fred Wagner, Dale Weisbrot, Bernard Wilhelm, Christopher Wilhelm, Janie Wilhelm, Pierre Wilhelm, Rita Wilhelm, Cheryl Zagozeski.

32. ROSETOWN-WISETON. Dec. 26:60 miles by car in 2 hours, $3-1 / 2$ miles on foot in 2 hours, temp. $25^{\circ}-30^{\circ}$, clear, I inch of 


\section{Species recorded from more than 1 locality on Count day}

\begin{tabular}{|c|c|c|c|c|c|c|c|c|c|c|c|c|}
\hline Mallard & & & & 5 & & & & & & & & 3 \\
\hline Pintail & & & & & & & & & & & & 2 \\
\hline Redhead & & & & 1 & & & & & & & & 2 \\
\hline Lesser Scaup & & & & & & & & & & & & 3 \\
\hline Common Goldeneye & & & & 25 & & & & & & & & 5 \\
\hline Goshawk & & & & & & & & & & & & 6 \\
\hline Rough-leg. Hawk & & & & & & & & & & & & 2 \\
\hline Golden Eagle & & & 2 & & & 1 & & & & & & 5 \\
\hline Bald Eagle & & & & 2 & & & & & & & & 5 \\
\hline Merlin & & & & & & & & & & & & 4 \\
\hline Spruce Grouse & & & & 1 & & & & & & & & 2 \\
\hline Ruffed Grouse & & & & 3 & & & & & & & & 11 \\
\hline Sharp-tailed Grouse & & 9 & & 1 & & 28 & & & & & 9 & 23 \\
\hline Ring-neck. Pheasant & & & & & & & & & & & & 3 \\
\hline Gray Partridge & & & 31 & & & & 8 & & 14 & & & 14 \\
\hline Rock Dove & & & 28 & & 8 & & 34 & & & & & 23 \\
\hline Mourning Dove & & & & & & & & & & & & 2 \\
\hline Great Horned Owl & & 1 & & & & & & & & 1 & 1 & 19 \\
\hline Snowy Owl & & 1 & 4 & & & & & & & & 3 & 18 \\
\hline Short-eared Owl & & & 1 & & & & & & 2 & & & 6 \\
\hline Common Flicker & & & & & & & & & & & & 2 \\
\hline Pileated Woodpecker & & & & 1 & & & & & & & & 2 \\
\hline Hairy Woodpecker & & 4 & & 1 & & & 3 & 2 & & & & 19 \\
\hline Downy Woodpecker & 1 & 7 & & 1 & & & 1 & & & & 2 & 21 \\
\hline Horned Lark & & & 4 & & & 6 & 26 & & & & & 14 \\
\hline Gray Jay & & & & 6 & & & & 3 & & & & 3 \\
\hline Blue Jay & & 2 & & 1 & & & & & 1 & & & 9 \\
\hline Black-billed Magpie & & 18 & 10 & 24 & 7 & 2 & 9 & & 7 & 1 & 33 & 41 \\
\hline Common Raven & 2 & 1 & & 21 & & & & 3 & & 8 & 2 & 12 \\
\hline Bl.-cap. Chickadee & 2 & 23 & & 8 & & & 8 & 2 & & 5 & & 30 \\
\hline Boreal Chickadee & & & & & & & & 2 & & & & 6 \\
\hline W-breasted Nuthatch & & 2 & & & & & & & & & & 5 \\
\hline R-breasted Nuthatch & & & & & & & 2 & & & & & 6 \\
\hline Brown Creeper & & & & & & & & & & & & 2 \\
\hline American Robin & & & & & & & & & & & & 2 \\
\hline Gold.-cr. Kinglet & & 2 & & & & & 5 & & & & & 12 \\
\hline Bohemian Waxwing & & 8 & & & & & & & & 16 & 9 & 17 \\
\hline Cedar Waxwing & & & & & & & & & & & & 3 \\
\hline Northern Shrike & & & & 1 & & & & & & & & 4 \\
\hline Starling & & & 5 & & & & & & & & & 9 \\
\hline House Sparrow & 42 & 214 & 263 & 12 & & & 310 & & & 6 & 505 & 37 \\
\hline West. Meadowlark & & & & & & & & & & & & 2 \\
\hline Rusty Blackbird & & & & & & & & & & & & 2 \\
\hline Evening Grosbeak & & 4 & & 17 & & & & & & 1 & & 8 \\
\hline Pine Grosbeak & & 3 & & 3 & & & & & & 4 & & 13 \\
\hline Hoary Redpoll & & & & & & & & & & & & 3 \\
\hline Common Redpoll & 6 & 91 & 4 & & & & 13 & 21 & & & 125 & 24 \\
\hline Red Crossbill & & & & & & & & & & & & 2 \\
\hline Dark-eyed Junco & & & & & & & & & & & & 3 \\
\hline Snow Bunting & 6 & & 70 & 1000 & 30 & & 142 & & & 50 & & 28 \\
\hline & & & & & & & & & & & & \\
\hline Single Species & & & & & & & 1 & 2 & 1 & & & \\
\hline
\end{tabular}

Single Species

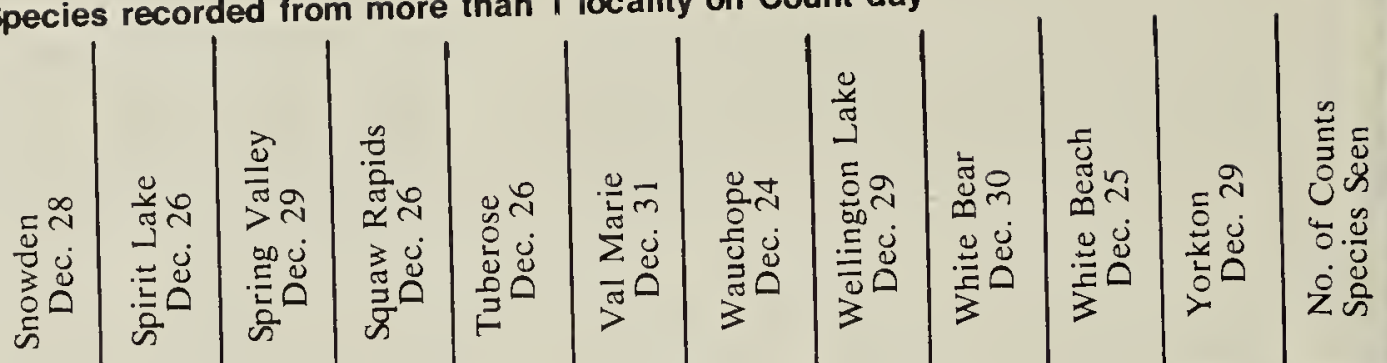


ow: 8 species, 377 individuals. - Alan, fian and Grev Jones, Chester Walker.

SASKATOON. Dec. 26; 300 miles by $r$ in 39-1/2 hours, 107 miles on foot in 67 urs, temp. $17^{\circ}-28^{\circ}$, wind W-SW 2-10 ph, clear, 1-2 inches of snow; 42 species, 82 individuals. (Add: Gadwall, 1, Jan. 1; own Creeper, 1, Dec. 25; White-winged ossbill, 3, Dec. 20). - Mark Abley, oira Atkinson, Bob, Garth, Jeff and Joyce esant, Bernard, Ed, Raymond and imothy Bisha, Ron Bobowski, Andre buthilette, Joe Daly, Alan, Edward, and artley Fredeen, Marie Gillespie, J.B. and adeleine Gollop, Jack Greaves, Scott ale, Bert Hardy, Wayne Harris, Jimmy effernan, Frank Hough, Dave, Don, ary, Stan and Stuart Houston, Ann and lark Jeffries, Sheila Lamont: Anna and ed Leighton, Robbie Mahood, Don and anne McRobbie, Aileen, Gray, Michael d Paul Merriam, Greg Michalenko, Anna
Miller, Arnold, Carmen and Ingrid Nijssen, Lynn Oliphant, Stuart Rasmussen, Don and Wayne Renaud, Adam and David Schmidt, John, Mary and Stan Shadick, Alan, Eric and Garry Smith, Doug Whitfield, Elmer Wright, Lucy Young.

34. SNOWDEN. Dec. $28 ; 7$ miles by car in $1-1 / 2$ hours, 4 miles on foot in 3 hours. temp. $20^{\circ}$, wind 15-20 mph., sunny, 8-10 inches of snow; 6 species, 59 individuals (Add: Snowy Owl, 1, Dec. 23). - Juliana Soroka.

35. SPIRIT LAKE. Dec. 26; 3 miles on foot in 3 hours, 54 miles by car in 4 hours, temp. $33^{\circ}$, wind W 15 mph, mostly clear, 5 inches of snow; 16 species, 390 individuals. (Add: Goshawk, 1, Dec. 21: Ruffed Grouse, 2, Dec. 27; Snow Bunting, 15, Dec. 21). - Bill and Joyce Anaka, Mrs. Gunn.

36. SPRING VALLEY. Dec. 29; 42 miles by car in $3-1 / 2$ hours, 8 miles on foot in 2 -

Species recorded from only 1 locality on Count day.

\section{No. Species}

Eared Grebe

Pied-billed Grebe

Mute Swan

Canada Goose

White-fronted Goose

Snow Goose

Ross's Goose

Gadwall

Green-winged Teal

American Wigeon

Northern Shoveler

Canvasback

Greater Scaup

White-winged Scoter

Ruddy Duck

Hooded Merganser

Sharp-shinned Hawk

Gyrfalcon

Prairie Falcon

Willow Ptarmigan

American Coot

Hawk Owl

Black-backed 3-toed Woodpecker

Northern 3-toed Woodpecker

Red-winged Blackbird

Common Grackle

White-winged Crossbill

Tree Sparrow

White-crowned Sparrow
Locality

Map No.

Regina

Regina

Regina

Regina

31

Saskatoon

Regina

Regina

Broadview

Saskatoon

Broadview

Saskatoon

Regina

33

Regina

Cold River

Regina

Regina

Big Gully

Regina

White Bear

Wellington Lake $\quad+1$

Regina 31

Wellington Lake $\quad+1$

Besnard Lake 3

Besnard Lake 3

Wauchope 40

Regina

Big Gully

Saskatoon

Saskatoon 
$3 / 4$ hours, around yard $3 / 4$ hour, temp. $10^{\circ}$ $22^{\circ}$, sunny, wind brisk, 8 inches of snow; 11 species, 422 individuals. (Add: Prairie Falcon, 1, Dec. 17; Sharp-tailed Grouse, 5, Dec. 20 and 6, Dec. 30; Great Horned Owl, 1, Dec. 27, 28; Northern Shrike, 1, Dec. 30). - Allan Bogdan, Flossie Bogdan (compiler), Larry Bogdan, Nick Bogdan.

37. SQUAW RAPIDS. Dec. 26; 7 hours in field, temp. $25^{\circ}$, overcast, no wind; 20 species, 1134 individuals. - Gladys and Stan Riome.

38. TUBEROSE-KYLE. Dec. 26; 64 miles by car and $1-1 / 2$ miles on foot in 3 hours, temp. $18^{\circ}$, wind SE $8 \mathrm{mph}$, clear; 3 species, 45 individuals. - Cliff Matthews.

39. VAL MARIE. Dec. 31; 50 miles by car and 2 miles on foot, temp. $28^{\circ}$, sunny, calm, 2 inches of snow; 4 species, 37 individuals. (Add: Prairie Falcon, 1, Dec. 24; Gray Partridge, 3, Dec. 26; Snowy Owl, 1, Dec. 29; Common Redpoll, 3, Dec. 24; Snow Bunting, 100, Dec. 26). - J. David Chandler.

40. WAUCHOPE. Dec. $24 ; 33-1 / 2$ miles $(53.6 \mathrm{~km})$ by car in 3 hours, 3 miles $(4.8$ $\mathrm{km}$ ) on foot in 3 hours, and watching feeder throughout day, temp. $-4^{\circ} \mathrm{F}$ to $22^{\circ} \mathrm{F}\left(-20^{\circ} \mathrm{C}\right.$ to $-6^{\circ} \mathrm{C}$ ), wind $\mathrm{SW} 3-4 \mathrm{mph}(4-5 \mathrm{~km})$ in P.M., overcast and heavy fog in A.M., clearing mid morning, then partial cloud cover, snow 10 inches $(28 \mathrm{~cm}) ; 13$ species, 562 individuals. (Add: Sharp-tailed Grouse, 4, Dec. 23; Ring-necked Pheasant, 1, Dec. 25; Evening Grosbeak, 11, Dec. 30). - Dale Hjertaas (compiler), Elinor Hjertaas, Warren Hjertaas.

41. WELLINGTON LAKE $\left(59^{\circ} 38^{\prime} \mathrm{N} 109^{\circ}\right.$ $02^{\prime}$ W) . Dec. 29 ; temp. $19^{\circ}$, calm: 8 species, 39 individuals. - Edith and Roy Middleton.

42. WHITE BEAR. Dec. 30; 5-1/2 miles by car, 3 miles on foot, temp. $30^{\circ}$, light west wind, sunny, light snow cover: 5 species, 25 individuals. (Add: Mourning Dove, 1, Dec. 26). - Carl and Peter Bischoff, Oran Cates, Leroy Clark, Douglas Gunn, Gary, Laine and Sig Jordheim, David Lowe, Dan Schuler (White Bear Conservation Club).

43. WHITE BEECH-ARRAN. Dec. $25 ; 65$ miles by car and at feeding station, 9 species, 92 individuals. - Margaret Hutchinson, Mr. and Mrs. L. A. Wotherspoon.

44. YORKTON. Dec. 29; 76 miles by car and 2 miles on foot, temp. $6^{\circ}$, sunny, wind $S$ $6 \mathrm{mph}, 5$ inches of snow; 9 species, 689 individuals. - Margaret Bromley (compiler), Lorne Lepp, Larry Morgotch, Phil Pawluck, Harold Wilkinson.

\section{FORT SMITH, N.W.T., CHRISTMAS BIRD COUNT}

by ERNIE KUYT*

Date: December 28, 1974.

Weather: Sunny and clear; tem perature $-18^{\circ} \mathrm{F}$; light wind; sno depth about $27 \mathrm{~cm}$; daylight 9:00 a.m - 4:00 p.m.

Routes covered: Fort Smith to Foll Holes road intersection; - Fort Smit along Pine Lake road to Salt Rive Fort Smith to Mountain Portage 0 cross-country skis. Total $80+48$ $12=140 \mathrm{~km}$. from $10-2$ p.m.

Birds seen: Raven 141, Gray Jay Rock Dove 45, Spruce Grouse Sharp-tailed grouse 8, Willow Pta migan 1, Hairy Woodpecker 1, Down Woodpecker 1, Pine Grosbeak 21 t Hoary Redpoll 23, Black-cappe Chickadee 4, House Sparrow 4.

Contributors: Elsie, Ernie, Pamel: Jonathan Kuyt; Linda and Da Graham; Hilah, Norman, David Sim mons: Debbie, Rex, Billie, Coupland: Alan Lee and Jim Mills.

*Box 508

Fort Smith, N.W.T.

\section{WATCH FOR MARKED COWBIRDS}

Over 7,100 Brown-headed Cowbirds were banded and colour marked in west-central Kansas during 1974 as an aid in studying their movements and, hopefully, to determine their places of origin Birds were marked with red, yellou or green plastic leg streamers. Fal and winter observations revealed 27 individuals from 10 locations it Kansas, Oklahoma, Texas and Mexico. However, data from spring and summer movements ar needed. Observers can help b. reporting location and date o sighting, sex of bird, and colour o leg streamer to Richard A. Hill Department of Biology, Fort Hay Kansas State College, Hays, Kansa 67601 . 